\title{
Acidentes com perfurocortantes envolvendo profissionais e estudantes da área de saúde: diagnóstico em um hospital universitário de referência
}

\author{
Maria Clara Domingos de Araújo Sousa \\ Acadêmica de Medicina, Universidade Estadual de Ciências da Saúde de Alagoas, Maceió, Al, Brasil \\ $\bowtie$ claradomingos1@hotmail.com \\ Claudio José dos Santos Júnior \\ Mestrando em Ensino na Saúde e Tecnologia. Biólogo. Acadêmico de Medicina, Universidade Estadual de \\ Ciências da Saúde de Alagoas, Maceió, Al, Brasil \\ 凶.laudiosantos@hotmail.com \\ Tarcísia Domingos de Araújo Sousa \\ Diretora-Presidente do Instituto Mineiro de Gestão de Águas - IGAM \\ $\triangle$ tarcisiadsousa@gmail.com \\ Guilherme Calixto dos Santos Neves \\ Acadêmico de Medicina, Universidade Estadual de Ciências da Saúde de Alagoas, Maceió, Al, Brasil. \\ $\triangle$ calixtoguilherme@outlook.com \\ Yáskara Veruska Ribeiro Barros \\ Mestre. Docente da Universidade Estadual de Ciências da Saúde de Alagoas, Maceió, Al, Brasil \\ \yaveriba@yahoo.com.br
}

Recebido em 20 de maio de 2020

Aceito em 26 de janeiro de 2021

\section{Resumo:}

O Brasil registra uma média de 700 mil acidentes ocupacionais por ano, ocupando o $4^{\circ}$ lugar no mundo em ocorrência de acidentes de trabalho. Dentre estes eventos, o mais frequente no meio hospitalar envolve os materiais perfurocortantes. O objetivo deste trabalho foi avaliar os acidentes com perfurocortantes envolvendo profissionais e estudantes da área da saúde atendidos em um Hospital de Referência no estado de Alagoas. Os dados foram obtidos da ficha de registro de acidentes de trabalho disponibilizada pelo Núcleo de Vigilância Epidemiologia da Unidade, sendo tabulados e analisados em termos de frequência absoluta e relativa e através de teste qui-quadrado de Yates para variáveis qualitativas nominais. O presente estudo constatou que dos 2.413 acidentados, 1859 (77,0\%) eram do sexo feminino, $1678(69,6 \%)$ possuíam idades entre $20-40$ anos, sendo a maioria, auxiliares e técnicos de enfermagem (44,2\%). Com relação aos acidentes ocorridos, $302(15,6 \%)$ foram durante procedimentos cirúrgicos e 61 (39,9\%) envolvendo acadêmicos de odontologia. Foi observado que durante os acidentes, $438(65,1)$ dos indivíduos não utilizavam luvas, 453 indivíduos $(18,8 \%)$ não foram vacinados contra a Hepatite B, 1462 sujeitos $(67,0 \%)$ tinham o paciente-fonte identificado. Foi possível verificar, também, que 819 acidentados $(71,2 \%)$ abandonaram o tratamento. Houve predomínio de grupos específicos, tanto na categoria profissionais de saúde quanto na categoria discentes, revelando maior susceptibilidade de técnicos/auxiliares de enfermagem e discentes de odontologia aos acidentes com material perfurocortante. Torna-se necessário, desse modo, o incentivo à adesão às precauções-padrão e ao uso de Equipamento de Proteção Individual (EPI) por meio da educação permanente e continuada no meio hospitalar, bem como a adequação das instituições às exigências de Normas Regulamentadoras de prevenção de acidentes laborais, objetivando a minimização dos riscos e de modo a oferecer um ambiente mais seguro.

Palavras-chave: Acidentes de trabalho, Exposição a agentes biológicos, Riscos ocupacionais. 


\title{
Sharps accidents involving health professionals and students: diagnosis at a reference university hospital
}

\begin{abstract}
:
Brazil records an average of 700 thousand occupational accidents per year, ranking 4 th in the world in terms of occupational accidents. Among these events, the most frequent in the hospital environment involves sharps. The objective of this work was to evaluate the accidents with sharps involving professionals and students of the health area treated at a Reference Hospital in the state of Alagoas. The data were obtained from the work accident registration form provided by the Unit's Epidemiology Surveillance Center, being tabulated and analyzed in terms of absolute and relative frequency and through the Yates chi-square test for nominal qualitative variables. The present study found that of the 2,413 accident victims, 1859 (77.0\%) were female, 1678 (69.6\%) were aged between 20-40 years, with the majority being nursing assistants and technicians (44.2\%). Regarding accidents, $302(15.6 \%)$ were during surgical procedures and 61 (39.9\%) involving dentistry students. It was observed that during accidents, 438 (65.1) of the individuals did not use gloves, 453 individuals (18.8\%) were not vaccinated against Hepatitis B, 1462 subjects $(67.0 \%)$ had the source patient identified. It was also possible to verify that 819 accident victims (71.2\%) abandoned the treatment. There was a predominance of specific groups, both in the health professional category and in the student category, revealing greater susceptibility of nursing technicians / assistants and dental students to accidents with sharp objects. It is therefore necessary to encourage adherence to standard precautions and the use of Personal Protective Equipment (PPE) through permanent and continuing education in the hospital environment, as well as the adaptation of institutions to the requirements of Regulatory Norms for prevention of occupational accidents, aiming at minimizing risks and in order to offer a safer environment.
\end{abstract}

Keywords: Accidents occupational, Exposure to biological agents, Occupational risk.

\section{Accidentes punzantes que involucran a profesionales de la salud y estudiantes: diagnóstico en un hospital universitario de referencia}

\begin{abstract}
Resumen:
Brasil registra un promedio de 700 mil accidentes laborales por año, ocupando el cuarto lugar en el mundo en términos de accidentes laborales. Entre estos eventos, el más frecuente en el entorno hospitalario involucra objetos punzantes. El objetivo de este trabajo fue evaluar los accidentes con objetos punzantes que involucran a profesionales y estudiantes del área de salud tratados en un Hospital de referencia en el estado de Alagoas. Los datos se obtuvieron del formulario de registro de accidentes de trabajo proporcionado por el Centro de Vigilancia de Epidemiología de la Unidad, que se tabuló y analizó en términos de frecuencia absoluta y relativa y a través de la prueba de chicuadrado de Yates para variables cualitativas nominales. El presente estudio encontró que de las 2,413 víctimas de accidentes, 1859 (77\%) eran mujeres, 1678 (69.6\%) tenían entre 20 y 40 años, y la mayoría eran auxiliares de enfermería y técnicos (44.2\%). Con respecto a los accidentes, $302(15,6 \%)$ fueron durante procedimientos quirúrgicos y $61(39,9 \%)$ con estudiantes de odontología. Se observó que durante los accidentes, 438 (65.1) de los individuos no usaron guantes, 453 (18.8\%) no fueron vacunados contra la hepatitis B, 1462 sujetos (67.0\%) identificaron al paciente fuente. También fue posible verificar que 819 víctimas de accidentes (71.2\%) abandonaron el tratamiento. Hubo un predominio de grupos específicos, tanto en la categoría de profesionales de la salud como en la categoría de estudiantes, revelando una mayor susceptibilidad de los técnicos / asistentes de enfermería y estudiantes de odontología a accidentes con objetos punzantes. Por lo tanto, es necesario fomentar el cumplimiento de las precauciones estándar y el uso de equipos de protección personal (EPP) a través de la educación permanente y continua en el entorno hospitalario, así como la adaptación de las instituciones a los requisitos de las normas reguladoras para prevención de accidentes laborales, con el objetivo de minimizar riesgos y ofrecer un ambiente más seguro. Palabras clave: Accidentes de trabajo, exposición a agentes biológicos, riesgos laborales.
\end{abstract}




\section{INTRODUÇÃO}

O trabalho desempenha papel fundamental nas atividades sociais de vida do homem (LIMA. et al., 2015). Apresenta-se como mecanismo para subsistência e como forma de realização humana, entretanto, quando não oferecer condições adequadas pode ser fonte de risco para a saúde dos envolvidos (REZENDE, et al., 2015).

De acordo com dados divulgados pela Organização Internacional do Trabalho (OIT) cerca de 2,3 milhões de pessoas morrem e outras 300 milhões são feridas todos os anos no mundo envolvendo-se em acidentes de trabalho. O Brasil ocupa a quarta posição com um total de 700 mil acidentes ocupacionais/ano, atrás somente de China, Índia e Indonésia (OIT, 2013).

A Lei $n^{\circ} 8.213 / 91$, em seu art. 19, define o acidente ocupacional como um evento que ocorre pelo exercício do trabalho, provocando lesão corporal ou alteração funcional que desencadeie a morte ou ainda a perda ou diminuição permanente ou temporária, da capacidade para a atividade (BRASIL, 2013).

Dentre os vários tipos de acidentes laborais, destacam-se os que envolvem materiais perfurocortantes e fluidos corporais, devido à realização de atividades com utilização, principalmente de agulha, mas também tesoura, lâmina de bisturi e outros instrumentais (OLIVEIRA; GONÇALVES, 2010; SILVA et al., 2010). A literatura descreve inúmeras causas relatadas pelos profissionais da saúde para a ocorrência dos acidentes com perfurocortantes (DIAS, 2014). Dentre estes, destacam-se descuido profissional e processo de descarte inadequado do material perfurocortante, além de A negligência com o devido uso dos Equipamentos de proteção individual (EPI), principalmente entre os acadêmicos (RODRIGUES et al., 2017).

A ocorrência desses acidentes repercute seriamente na saúde dos profissionais (RODRIGUES et al., 2017). De modo a gerar prejuízos físicos e psicológicos, aumentando o risco de transmissão de doenças infectocontagiosas (como Hepatite B, Hepatite C e infecção pelo HIV), provocando sentimento de culpa e transtorno para trabalhadores e pacientes, de maneira a impactar a saúde como um todos dos profissionais que prestam os serviços de saúde (CÂMARA et al., 2011).

Outra problemática envolvida nos acidentes são as subnotificações das ocorrências, de modo a revelar uma pouca procura por atendimento médico após acidente (PAIVA et al., 2017; LOPES et al., 2017). Dentre os vários motivos relatados pelos profissionais da área da 
saúde, destacam-se a irrelevância do acidente, desconhecimento do protocolo de rotina, displicência e sobrecarga de trabalho(OLIVEIRA et al., 2015).

Torna-se evidente a necessidade de maiores produções científicas acerca dos acidentes com materiais biológicos em profissionais de saúde, visto que é preciso identificar que lacunas ainda existentes (LOPES et al., 2017).

O ambiente hospitalar esconde riscos iminentes e diversas estratégias são necessárias para prevenir os acidentes de trabalho (FERREIRA et al., 2012). Diante disso, torna-se imprescindível a análise situacional dos acidentes laborais ocorridos nos hospitais de uma região. Segundo Anuário Estatístico da Previdência Social do Brasil, esses eventos, em sua maioria, envolvem materiais perfurocortantes (BRASIL, 2018).

Com a identificação dos principais fatores propiciadores dos acidentes é possivel proporcionar mudanças na formação dos profissionais e na sua rotina professional, tornandoos mais qualificados e aptos a atuarem de forma correta e com mais segurança na realização de suas atividades (COUTO, 2018; DOMBROSKI, 2018).

\section{MÉTODOS}

\section{Delineamento}

Estudo transversal, quantitativo e descritivo, do tipo documental.

\section{Local, população e amostra}

O Hospital Escola Dr. Helvio Auto (HEHA) trata-se de uma unidade de saúde de referência para o estado de Estado de Alagoas e seus 102 municípios e realiza o atendimento de moléstias infectocontagiosas e suas complicações. Constitui, também, em uma unidade de referência para acolhimento para os casos de acidentes ocupacionais no âmbito estadual.

Foram incluídos neste estudo todos os casos acidentes ocupacionais envolvendo perfurocortantes, com profissionais ou estudantes da área da saúde, atendidos no HEHA, no período de janeiro de 2012 a dezembro de 2017. 
Foram excluídos os registros que não continham a informação "tipo de exposição", "ano de acidente" e aqueles casos de indivíduos acidentados cuja profissão não estava vinculada à área da saúde, com exceção dos trabalhadores pertencentes a equipe de limpeza.

Totalizaram-se inicialmente 2.443 casos de acidentes ocorridos com trabalhadores e estudantes da área da saúde, envolvendo materiais perfurocortantes, no período de 01/01/2012 a 31/12/2017. Entretanto, após aplicação dos critérios de inclusão e exclusão acima explicitados obteve-se um total de 2.413 casos avaliados.

Os dados foram obtidos através do registro de acidentes do Núcleo de Vigilância Epidemiológica da Unidade (NVE/HEHA) que processou as informações contidas na ficha de comunicação de acidente de trabalho (CAT). Tais dados foram tabulados eletronicamente no programa Tabwin (DATASU/MS), garantindo a segurança do sigilo de identificação dos sujeitos da pesquisa.

\section{Análise dos dados}

Neste estudo, foram avaliadas as seguintes variáveis: sexo, idade, ocupação, tipo de exposição, circunstância do acidente, agente causador, uso de equipamento de proteção individual (EPI), situação vacinal do acidentado, conhecimento ou não sobre o paciente fonte e evolução.

Todos os dados foram digitados, processados, tabulados e analisados no software de estatística BioEstat 3.0. As variáveis qualitativas nominais foram analisadas através do teste qui-quadrado de Yates e as qualitativas ordinais através de estatística descritiva de frequência de frequência absoluta e relativa.

\section{Aspectos Éticos}

Esta pesquisa foi submetida ao Comitê de Ética em Pesquisa (CEP) da Universidade Estadual de Ciências da Saúde de Alagoas (Uncisal) com o CAAE nº 69805417.9.0000.5011 e parecer consubstanciado de aprovação nº 2.132.909.

A coleta dos dados somente foi iniciada após aprovação do CEP. 


\section{RESULTADOS}

$\mathrm{Na}$ investigação dos acidentes registrados, foi constatada a ocorrência de 2.413 acidentes de trabalho com materiais perfurocortantes envolvendo profissionais e acadêmicos da área de saúde.

Neste trabalho optou-se por apresentar os resultados em dois eixos, sendo um deles acerca das características demográficas e ocupacionais dos acidentados e outro referente à caracterização do evento de interesse.

\section{Características demográficas e ocupacionais dos acidentados}

Em relação ao sexo, tem-se a predominância de indivíduos do sexo feminino, correspondendo a 77\% (1.859) dos acidentados, enquanto que apenas 23\% (554) das pessoas do sexo masculino estão expressos na amostra.

Com relação à faixa etária, a idade mínima dos expostos foi de 17 anos e a idade máxima, 85 anos. A idade média observada foi de 33,5 anos. Foi verificado, ademais, uma predominância de acidentados em idade produtiva, entre 20 e 40 anos, grupo que correspondeu de a 69,6\% (1.678) do total de acidentados.

A parcela mais expressiva dos profissionais acidentados correspondeu à classe dos auxiliares/técnicos de enfermagem, compreendendo 54,2\% (1.070) dos casos, seguida pela equipe de limpeza com $16,1 \%$ (317), enfermeiros $(8,4 \% ; 165)$ e profissionais dentistas $(7,5 \%$; 149).

Dentre os acadêmicos, discentes do curso de odontologia somaram 39,9\% (61) do total de casos analisados, seguido pelos acadêmicos de medicina com 30,1\% (46), enfermagem 20,9\% (32) e pelos estudantes que realizam curso técnico na área de saúde (7,8\%).

Na Tabela 1 apresentamos a síntese das características demográficas e ocupacionais dos acidentados que fizeram parte deste trabalho. 
Tabela 1 - Características demográficas e ocupacionais dos acidentados.

\begin{tabular}{lllllll}
\hline Características & $\mathrm{N}$ & $\%$ & $\mathrm{X}^{2}$ & $p$ \\
\hline
\end{tabular}

$\operatorname{Sexo}(N=2.413)$

Feminino

$1.859 \quad 77$

Masculino

55423

$28,0<0.0001$

Idade (anos) $(\mathrm{N}=2.413)$

Média / DP

Variação

$<20$ anos

$20-40$ anos

$>40$ anos

Profissionais $(\mathrm{N}=1.974)^{*}$

Auxiliar/técnico de enfermagem

Equipe de limpeza

Enfermeiro

Odontologista

Médico

Auxiliar/técnico de saúde bucal

Auxiliar/técnico de laboratório

Fisioterapeuta

Acadêmicos $(\mathrm{N}=153)^{*}$

Odontologia

Medicina

Enfermagem

Técnico (enfermagem, laboratório ou saúde bucal)

Fisioterapia
$33,5 / 10,6$

17-85

$\begin{array}{cc}93 & 3,8 \\ 1678 & 69,6 \\ 642 & 26,6\end{array}$

1070

54,2

$317 \quad 16,1$

$165 \quad 8,4$

$149 \quad 7,5$

$132 \quad 6,7$

$170,5<0.0001$

$57 \quad 2,9$

$53 \quad 2,7$

$31 \quad 1,6$

$61 \quad 39,9$

$46 \quad 30,1$

$32 \quad 20,9 \quad 49,8 \quad<0.0001$

$12 \quad 7,8$

$2 \quad 1,3$

Legenda: *missing: 286 notificações de acidentes com profissionais de saúde não faziam distinção entre categoria (estudante e/ou profissional) e não especificavam a área de atuação. $\mathrm{X}^{2}$ teste qui-quadrado de Yates.

Fonte: Acervo documental do Projeto (2019). 


\section{Caracterização dos acidentes de trabalho}

Quanto à situação de ocorrência do acidente ocupacional, constatou-se que a maioria dos casos ocorreu em procedimentos cirúrgicos, totalizando 15,6\% (302) dos eventos, sendo esse número sucedido do processo de administração de medicação endovenosa 12,8\% (263) e do descarte inadequado de material pefurocortante em bancada, cama, chão e afins (10,9\%; 225). A menor representação percentual encontra-se nos processos de administração de medicação intradérmica e re-encape, correspondendo, respectivamente, a 1,9\% (39) e 1,7\% (35) dos casos. É viável destacar que alguns acidentes não tiveram o item "circunstância do acidente" preenchido, de modo a serem classificados como "ignorado" que corresponde a 358 casos $(14,8 \%)$ de todos os casos.

O principal agente causador foi a agulha com lúmen (luz) responsável por 72,6\% (1.674) dos eventos, seguida da agulha sem lúmen/maciça com 10,3\% (237).

Quanto ao uso dos Equipamentos de Proteção Individual (EPI) apenas 65,1\% (1.570) dos acidentados afirmam fazer uso de luva no momento do acidente, 25,1\% (605) utilizavam avental, 3\% (72) proteção facial, 16,7\% (402) máscara e 11,6\% (281) usavam óculos.

A situação vacinal irregular do acidentado frente à Hepatite B foi observada em 18,8\% (453) dos indivíduos acidentados.

O paciente-fonte foi identificado em 67\% (1462) dos casos. Como evolução o abandono foi a principal vertente observada representando $71,2 \%$ (819) dos casos.

Tabela 2 - Características dos acidentes com material pefurocortante.

\begin{tabular}{|c|c|c|c|c|}
\hline Características & $\mathrm{N}$ & $\%$ & $\mathrm{X}^{2}$ & $p$ \\
\hline \multicolumn{5}{|l|}{ Circunstância do acidente $(\mathrm{N}=2.055)$} \\
\hline Procedimento cirúrgico & 320 & 15,6 & \multirow{6}{*}{32,1} & \multirow{6}{*}{0,023} \\
\hline Administração de medicação endovenosa & 263 & 12,8 & & \\
\hline Descarte inadequado de MPC em bancada, cama, chão e afins & 225 & 10,9 & & \\
\hline Outras situações & 198 & 9,6 & & \\
\hline Descarte inadequado de material perfurocortante em saco de lixo & 193 & 9,4 & & \\
\hline Manipulação de caixa com material perfurocortante & 167 & 8,1 & & \\
\hline
\end{tabular}


Administração de medicação intramuscular

153

Procedimento odontológico

$126 \quad 6,1$

Administração de medicação subcutânea

$108 \quad 5,3$

Punção venosa/arterial para coleta de sangue

$98 \quad 4,8$

Lavagem de material

Procedimento laboratorial

$76 \quad 3,7$

Administração de medicação intradérmica

Re-encape

Agente $(\mathrm{N}=2.306)$

Agulha com lúmen

$1674 \quad 72,6$

Agulha sem lúmen/maciça

$237 \quad 10,3$

Outros

Lâmina/lanceta (qualquer tipo)

$206 \quad 8,9$

143

6,2

Vidros

$29 \quad 1,3$

Intracath

Uso de EPI no momento do acidente $(\mathrm{N}=2.413)$

Luva

$1570 \quad 65,1$

Avental

$605 \quad 25,1$

Máscara

Óculos

$402 \quad 16,7$

$281 \quad 11,6$

$117,0<0.0001$

Bota

$200 \quad 8,3$

Proteção facial

723,0

Situação vacinal em relação à Hepatite B (3doses) $(N=2.413)$

Vacinado

$1822 \quad 75,5$

Não vacinado

$453 \quad 18,8$

$32,9<0.0001$

Conhecimento sobre o paciente-fonte $(N=2.413)$

Identificado

Não identificado

146267,0

71933,0

$10,89<0.0001$

Evolução (N=1.151)

Abandono

81971,2

Alta paciente fonte negativo

$250 \quad 21,7$

Alta sem conversão sorológica

79

6,9

$123,3<0.0001$

Alta com conversão sorológica

$3 \quad 0,3$

Legenda: *a diferença do $\mathrm{N}$ por variável e do total amostral (2.413) se refere ao conjunto de itens ignorados e/ou não preenchidos. $\mathrm{X}^{2:}$ teste qui-quadrado de Yates.

Fonte: Acervo documental do Projeto (2019). 


\section{DISCUSSÃO}

O presente estudo, com relação à variável sexo entre os acidentados, revelou uma predominância do sexo feminino sobre o masculino, fato observado no estudo de Santos Júnior et al. (2015), que apresentou em sua amostra cerca de 70\% de pessoas do sexo feminino, entre servidores e estudantes do em um Hospital de Referência da cidade Araguaína, Tocantins. Este fato é justificado, segundo Lopes et al. (2017) e Rodrigues et al. (2017) devido a equipe de enfermagem, que é geralmente mais acometida por esse tipo de acidente laboral, ser majoritariamente feminina.

Quanto à distribuição dos profissionais, a presente pesquisa corrobora com a literatura, segundo Dombroski (2018), ao obter a equipe de técnicos de enfermagem como os profissionais mais acometidos. Entretanto diverge de estudos como o de Santos Júnior et al. (2015) e Oliveira e Gonçalves (2010), os quais revelaram os médicos ocupando o segundo lugar entres os profissionais, diferentemente, do presente estudo, que aponta os profissionais da equipe de limpeza. Esse fato, de acordo com Oliveira et al. (2015), deve-se a subnotificação por parte de determinados profissionais, principalmente os de baixa escolaridade, relatarem medo de serem afastados do emprego ou terem perdas salariais e de benefícios.

Em relação à área estudantil mais suscetível, encontra-se, de forma quase unânime na literatura, os acadêmicos de odontologia, os quais, segundo Paiva et al. (2017) tem altos riscos devido ao fato de apresentarem um maior acúmulo de horas clínicas e, sobretudo, um maior contato com os materiais perfurocortantes logo nos primeiros anos de faculdade.

Com relação à circunstância e o agente mais comum envolvido nos acidentes, a pesquisa apresenta concordância com a literatura, a exemplo de Lima et al. (2015), o qual apontam o procedimento cirúrgico, que apresenta dedicação por extensas horas de trabalho, e a agulha com lúmen, pela dificuldade dos profissionais na aplicação de medidas de segurança, como os principais envolvidos.

Quanto ao uso de EPI no momento do acidente e a situação vacinal do acidentado revelaram dados alarmantes, que corroboram com a literatura, referentes a negligência e a ausência de informação adequada sobre segurança no trabalho por determinados profissionais e estudantes. Segundo resultado dos estudos de Cunha (2009) e Valin (2011), este primeiro realizado através da percepção de segurança e medidas preventivas contra os 
acidentes de profissionais atuantes na emergência de num hospital público do Rio de Janeiro, ficou evidente o parcial, e às vezes, o total desconhecimento sobre normas obrigatórias de biossegurança. Pode-se perceber também que apesar da alta porcentagem de indivíduos vacinados $(75,5 \%)$ na presente pesquisa, existe outros estudos no país que possuem índices maiores de vacinação contra Hepatite B, a exemplo o Lima et al. (2015), o qual revelou um índice de $83 \%$.

Sobre a evolução desses pacientes, a alta taxa de abandono encontrada na pesquisa, cerca de $71,2 \%$, corrobora com a literatura, chegando até ser maior em outros grupos como os dos acadêmicos de odontologia de uma universidade pública do noroeste paulista, que no trabalho de Garbin, Wakayama e Garbin (2016) chegaram a obter uma taxa de abandono de $35,7 \%$, ou seja, não realizaram os exames com a frequência recomendada pelo protocolo para acidentes com perfurocortantes. De modo a demonstrar que existe falhas quanto a orientação na área de biossegurança para esses indivíduos que prestam assistência direta ao paciente e, portanto, necessitam de condições seguras de trabalho.

\section{CONCLUSÃO}

As evidências encontradas nesse estudo indicam que auxiliares e técnicos de enfermagem, profissionais do sexo feminino, com idade entre 20 a 40 anos, são os indivíduos mais acometidos por acidentes com materiais perfurocortantes e que, dentre os estudantes, os acadêmicos de odontologia foram os mais acometidos.

Diante desse preocupante risco ocupacional para profissionais e estudantes da área da saúde, torna-se evidente a necessidade de orientação quanto ao uso adequado de medidas de proteção individual e coletiva e quanto às condutas que devem ser tomadas em casos de acidentes envolvendo os agentes estudados.

Importante destacar, por fim, que o desenvolvimento de pesquisas dessa natureza pode proporcionar mudanças na formação e na rotina de trabalho de profissionais e estudantes, adotadas no âmbito da gestão dos serviços ou pela adoção de medidas 
autocuidado e de maior vigilância, ambas com fim de minimizar riscos e de proporcionar uma atenção à saúde laboral mais segura.

\section{REFERÊNCIAS}

BRASIL. Conceito, definições e caracterização do acidente do trabalho: Prestações e procedimentos. Brasília: Ministério Da Previdência Social, 2013.

BRASIL. Secretaria de Previdência. Anuário Estatístico da Previdência Social (AEPS) 2017. Brasília: Ministério da Previdência Social, 2018.

CÂMARA, P. F.; LIRA, C.; SANTOS JUNIOR, B. J.; VILELLA, T. A. S.; HINRICHSEN, S. L. Investigação de acidentes biológicos entre profissionais da equipe multidisciplinar de um hospital. Revista de Enfermagem da UERJ, Rio de Janeiro, v. 19, n. 4, p. 583-586, 2011.

COUTO, P. L. S. Representações sociais acerca dos riscos de acidentes de trabalho. Revista Brasileira de Promoção da Saúde, v. 31, n. 2, p. 1-10, 2018.

CUNHA, A. C.; VALENTE, G. S. C. Desvelando o conhecimento dos trabalhadores de enfermagem acerca dos riscos biológicos na emergência. Ensino, Saúde e Ambiente, v. 2 n. 2 p. 69-83, 2009.

DIAS, A. Acidentes com material perfurocortante em profissionais da saúde: uma revisão de artigos indexados na biblioteca Virtual em saúde 2003-2013. 2014. 34 p. Dissertação (Saúde Pública) - Universidade Federal do Rio Grande do Sul, Departamento de Medicina Social, Porto Alegre, 2014.

DOMBROSKI, M. Avaliação de acidentes: riscos envolvendo material biológico e perfurocortantes. 2018. 58 p. Dissertação (Engenharia de Segurança do Trabalho) - Universidade do Sul de Santa Catarina, Florianópolis, 2018.

FERREIRA, A. F.; BRAGA E. S.; FERREIRA, A. F.; TELLES, F. L. Manejo Seguro de perfurocortantes: abordagem de acidentes em serviços de coleta. Revista Teccen, v. 10, n. 1, p. 24-30, 2017.

FERREIRA, H. F G.; PEREIRA, G. C.; NOGUEIRA, J. K. A; FERREIRA, M. R.; TEIXEIRA, D. G. Prevalência ocupacional de acidentes biológicos com material perfurocortante entre profissionais da saúde em âmbito hospitalar. Revista Mineira de Ciências da Saúde, v. 4, p. 78-87, 2012.

GARBIN, A. J. I.; WAKAYAMA, B.; GARBIN, C. A. S. Negligência no autocuidado em saúde: a imunização contra a Hepatite B na Odontologia. Archives of Health Investigation, v. 5, n. 2, p. 85-9, 2016.

LIMA, I. A. S.; OLIVEIRA, G. G.; RODRIGUES, A. R. G.; SOUSA, M. N. A. Acidentes Ocupacionais com Perfurocortantes: Estudo com Profissionais de Enfermagem. Revista Interdisciplinar em Saúde, v. 2, n. 1, p. 
Acidentes com perfurocortantes envolvendo profissionais e estudantes

da área de saúde: diagnóstico em um hospital universitário de referência

26-43, jan./mar. 2015.

LOPES, J. S. P.; CARVAlHO, T. E. S.; NASCIMENTO, J. F.; ALVES, C. A. S., PEREIRA, A. K. P., RODRIGUES, T. S. Características dos acidentes de trabalho com material biológico em profissionais de enfermagem. Revista Eletrônica Acervo Saúde, v. 9, n. 3, p. 1178-1186, 2017.

OLIVEIRA, A.; GONÇALVES, J. Acidente ocupacional por material perfurocortante em profissionais de saúde de um Centro Cirúrgico. Revista da Escola de Enfermagem USP, São Paulo, v.44, n. 2, p.482-487, 2010.

OLIVEIRA, J. S.; NERY, A. A.; MORAIS, R. L. G. L.; ROBAZZI, M. L. C. C. Acidentes com perfurocortante entre trabalhadores de saúde. Revista de APS, v. 18, n. 1, p. 108-115, 2015.

ORGANIZAÇÃO INTERNACIONAL DO TRABALHO (OIT). The prevention of occupational diseases. Genebra: OIT, 2013.

PAIVA, S. N.; ZARONI W. C. S.; LEITE, M. F.; BIANCHI, P. R.; PEREIRA, T. C. R. Acidentes ocupacionais com material biológico em Odontologia: uma responsabilidade no ensino. Revista da ABENO, v. 17, n. 3, p. 76-88, 2017.

REZENDE, L. C. M.; LEITE, K. N. S.; SANTOS, S. R.; MONTEIRO, L. C.; COSTA, M. B. S.; SANTOS, F. X. Acidentes de trabalho e suas repercussões na saúde dos profissionais de Enfermagem. Revista Baiana de Enfermagem, v. 29, n. 4, p. 307-317, 17 dez. 2015.

RODRIGUES, P. S.; SOUSA, A. F. L.; MAGRO, M. C. S.; ANDRADE, D.; HERMANN, P. R. S. Acidente ocupacional na Enfermagem. Escola Anna Nery, v. 21, n. 2, 2017.

SANTOS JÚNIOR, E. P.; BATISTA, R. R. A. M; ALMEIDA, A. T. F; ABREU, R. A. A. Acidente de trabalho com material perfurocortante envolvendo profissionais e estudantes da área da saúde em hospital de referência. Revista Brasileira de Medicina do Trabalho, v. 13, n. 2, p. 69-75, 2015.

SILVA, T.; ROCHA, S. A.; AYRES, J. A.; JULIANI, C. M. C. M. Acidente com material perfurocortante entre profissionais de enfermagem de um hospital universitário. Revista Gaucha de Enfermagem, Porto Alegre, v. 31, n. 4, p. 615-22, 2010

VALIM, M. D.; MARZIALE, M. H. P. Avaliação da exposição ocupacional a material biológico em serviços de Saúde. Texto e Contexto Enfermagem, v. 20, p.138-146, 2011.

\section{(cc) Br}

Este trabalho está licenciado com uma Licença Creative Commons - Atribuição 4.0 Internacional. 